\title{
Near Complete Response in a Patient with Classical Hodgkin Lymphoma Treated with Brentuximab Vedotin Concurrent with Radiation Therapy
}

\author{
Wilbur Montana ${ }^{a}$ Dennis Andrew Buck ${ }^{a}$ Tristan Smith ${ }^{b}$ \\ a Southwestern Regional Medical Center, Cancer Treatment Centers of America, \\ Tulsa, OK, USA; ${ }^{b}$ Oklahoma State University - Tulsa, Tulsa, OK, USA
}

\section{Keywords}

Brentuximab · Concurrent radiation · Classical Hodgkin lymphoma

\section{Abstract}

Brentuximab vedotin, an antibody drug conjugate that delivers monomethyl auristatin $E$ into CD-30 expressing cells is FDA approved for the treatment of patients with Hodgkin lymphoma after the failure of autologous stem cell transplantation or at least 2 prior multi-agent chemotherapy regiments. This approval was based on a study that showed an overall response rate of $75 \%$ and complete remission in $34 \%$. We present a case of a 24 -year-old male with classical nodular sclerosing Hodgkin lymphoma who achieved near complete remission following 5 cycles of brentuximab concurrent with ISRT (involved site radiation therapy) following progression of first-line ABVD (Adriamycin, bleomycin, vinblastine, dacarbazine) and subsequent second-line ICE (ifosfamide, carboplatin, etoposide) chemotherapy. This case not only reiterates the efficacy of brentuximab vedotin in the third-line setting but introduces the role of and need for further clinical trials of combined radiotherapy with brentuximab in Hodgkin lymphoma patients following failure of second-line options. 
 Oncology}

\section{Introduction}

CD30, also known as TNFRSF8, is a cell membrane protein of the tumor necrosis factor receptor family. It is expressed on the surface of Hodgkin/Reed-Sternberg cells in various tumor types, including Hodgkin lymphoma. Preliminary data have shown that anti-CD30 antibodies by themselves have limited response [1]. Brentuximab vedotin (BV) has the antitubulin monomethyl auristatin E conjugated to the CD30-specific monoclonal antibody, enhancing tumor activity [2]. The mechanism of action involves binding to the CD30, allowing the conjugate to be taken into the lysosomes, and thus allowing monomethyl auristatin E to be released leading to cell cycle arrest and subsequent apoptosis.

The FDA approved the use of BV (1.8 mg/kg dose every 3 weeks) based on a phase 1 trial showing a statistically significant overall response rate (ORR) and complete response (CR) in 102 patients with Hodgkin lymphoma and 58 patients with systemic anaplastic large cell lymphoma. The study patients had already received a median of 3 previous chemotherapy regimens, and $73 \%$ had undergone autologous stem cell transplantation. The results showed an ORR of $73 \%$ and a CR rate of 32\%. Based on this, the FDA granted approval of BV for the treatment of patients with Hodgkin lymphoma after the failure of autologous stem cell transplantation or at least 2 prior multi-agent chemotherapy regimens [3]. In a subsequent phase 2 trial, involving only patients with relapsed or refractory Hodgkin lymphoma, similar results were seen. The ORR was $75 \%$, with complete remission in $34 \%$ of patients [4]. A 5year follow-up end-of-study result from that trial demonstrated that a subset of these patients remained in remission, obtaining CR with BV, and may now be potentially cured [5]. Furthermore, even with relapse after achieving complete or partial remission with initial BV therapy, retreatment with BV monotherapy is still associated with a $68 \%$ response rate [6]. In addition to the above therapeutic efficacy and usage of brentuximab in refractory Hodgkin lymphoma, it has also been postulated that BV may also enable a more successful reducedintensity allogeneic hematopoietic cell transplantation in patients with relapsed or refractory Hodgkin lymphoma status after previous autologous transplantation $[7,8]$. These therefore highlight the important role that BV plays in the second- and/or third-line setting for refractory Hodgkin lymphoma.

However, the role of radiation in combination with BV in patients with Hodgkin lymphoma has not been adequately studied, largely due to concern for exacerbation of toxicities. While the most common side effects associated with BV are peripheral neuropathy and neutropenia $[9,10]$, the potential for pulmonary toxicity when combined with radiation therapy is of greatest concern. An early phase 1 study combining BV with ABVD (Adriamycin, bleomycin, vinblastine, dacarbazine) and/or gemcitabine in early-stage Hodgkin lymphoma had to be terminated prematurely due to the development of grade 3-5 pneumonitis [11]. It has been hypothesized that BV may be associated with subclinical pulmonary toxicity that is only manifested when an added risk for pulmonary toxicity is introduced, such as radiation therapy or bleomycin [12].

Nonetheless, a multicenter pilot study performed at Memorial Sloan-Kettering Cancer Center (MSKCC) and Wilmot Cancer Center sought to ascertain the safety and efficacy of combining BV with AVD (Adriamycin, vinblastine, dacarbazine) chemotherapy followed by ISRT (involved-site radiation therapy) in untreated early-stage classical Hodgkin lymphoma with unfavorable risk factors. Results of that study showed that all patients who completed BV + AVD + ISRT achieved a CR of $100 \%$. All patients tolerated the ISRT well and only grade 1-2 toxicities were reported [13]. Nonetheless, a major limitation of the study was the small 
sample size, which was adequate for the assessment of toxicity but not for efficacy assessment [13].

\section{Case Presentation}

We present the case of a 24-year-old Caucasian male with stage IVB progressive classical nodular sclerosing Hodgkin lymphoma, with disease affecting the anterior mediastinum with left supraclavicular extension, left peritracheal, left perihilar, pulmonary, and splenic involvement. The patient initially presented to our facility with progression of disease following first-line AVD systemic chemotherapy (bleomycin was omitted by prior oncologist due to pneumonia at initial presentation). He had a partial response upon interim PET/CT. However, after 6 cycles restaging PET/CT showed progression of disease with increased anterior mediastinal adenopathy and extensive activity with recurrent active disease extending into the left upper lobe of the lung (Fig. 1,2). The outside treating facility offered autologous stem cell transplantation or additional chemotherapy, which he declined. They had attempted to provide BV as second-line treatment, but this was denied by his insurance. He was not initially offered radiation therapy as a single modality due to the fact that that he had essentially primary refractory Hodgkin Lymphoma or at best a very rapid relapse. Nonetheless, he underwent radiation therapy to the neck and chest at an outside facility - 36 Gy over 20 fractions. A restaging PET/CT, however, again showed progressive disease with increased consolidation and nodularity in the lungs, new pulmonary nodules, and new lytic lesions in multiple left-sided ribs. Ultimately, patient was lost to follow-up until presentation at our facility.

Based on the fact that his status progressed after primary AVD, it was planned for him to be treated with second-line ICE (ifosfamide, carboplatin, etoposide) chemotherapy. However, after 2 cycles of ICE, the patient presented with B symptoms and complaints of worsening dyspnea at rest. Restaging PET/CT (status after 2 cycles of ICE) showed similar disease with a score of 5 on Deauville criteria (Fig. 3).

Our patient was then approved for third-line BV following the failure of 2 lines of standard treatment. At that time, he also underwent radiation, provided between doses of $\mathrm{BV}$, to the left chest wall and thoracic spine - 36 Gy TOMO-IMRT.

At 5 weeks following completion of 4 cycles of $\mathrm{BV}$, as well as radiation to the chest wall and thoracic spine, the PET/CT identified near CR with only a small (approx. $1 \mathrm{~cm}^{2}$ ) radiographically appearing avid residual lesion remaining in the region of the left lateral chest wall (Fig. 4). To date, the patient has denied any complaints of dyspnea or further symptomatology concerning pulmonary toxicity during BV or since (note: he was not provided bleomycin).

\section{Discussion}

Our patient presented with classical Hodgkin lymphoma with unfavorable features and his status progressed after 2 lines of conventional chemotherapy (AVD followed by ICE). He had previously declined autologous bone marrow transplantation with an outside facility. However, after 4 cycles of full-dose BV $(1.8 \mathrm{mg} / \mathrm{kg}$ dose every 3 weeks $)$ a near CR was seen. This clinical result corroborates with the findings a previous study by Younes et al. [14] with 
patients who had progressed on 2 previous lines of standard chemotherapy and/or who were not candidates for bone marrow transplantation.

Our patient, however, also received curative doses of concurrent radiation therapy interspersed between the doses of BV. A preliminary study has shown safety with regard to ISRT status after BV and AVD in newly diagnosed, previously untreated early-stage classical Hodgkin lymphoma with unfavorable features [13]. Although our patient had bulky disease with progression on 2 previous lines of chemotherapy, this case argues for further clinical trials in this subset of patients regarding efficacy of concurrent radiation with $\mathrm{BV}$, as our patient achieved an impressive near CR.

In the previous study by Younes et al. [14] regarding BV in patients previously progressing on 2 lines of therapy, the CR was only $32 \%$. Thus far, our team has not identified studies attempting to ascertain the potential benefit of adding concurrent radiation to BV. Though the MSKCC and Wilmot Cancer Center pilot study evaluated ISRT + BV + AVD, their sample size limited the ability to establish efficacy.

\section{Conclusion}

BV has been shown to induce an overall response and even complete remission in patients with classical Hodgkin lymphoma who previously failed 2 lines of standard chemotherapy and/or who were not candidates for stem cell transplantation [15]. ISRT in combination with BV may induce an improved CR in this patient population. Further studies need to be implemented to confirm combined modality treatment options whether it be the approach of the MSKCC and Wilmot Cancer Center (ISRT + BV + AVD) or the simplified approach in our case (ISRT + BV).

\section{Statement of Ethics}

The authors have no ethical conflicts to declare.

\section{Disclosure Statement}

The authors have no conflicts of interests to disclose.

\section{References}

1 Doronina SO, Toki BE, Torgov MY, et al: Development of potent monoclonal antibody auristatin conjugate for cancer therapy. Nat Biotechnol 2003;21:778-784.

2 Francisco JA, Cerveny CJ, Meyer DL, et al: cAC10-vcMMAE, an anti-CD30 monomethyl auristatin E conjugate with potent and selective antitumor activity. Blood 2003;102:1458-1465.

- 3 De Claro A, McGinn K, Kwitkowski V, et al: US Food and Drug Administration approval summary: brentuximab vedotin for the treatment of relapsed Hodgkin lymphoma or relapsed systemic anaplastic large cell lymphoma. Clin Cancer Res 2012;18:5845-5849.

-4 Younes A, Gopal A, Smith SE, et al: Results of a pivotal phase II study of brentuximab vedotin for patients with relapsed or refractory Hodgkin's lymphoma. J Clin Oncol 2012;30:2183-2188.

5 Chen R, Gopal, A, Smith S, et al: Five year survival and durability results of brentuximab vedotin in patients with relapsed or refractory Hodgkin lymphoma. Blood 2016;128:1562-1566. 


\section{Case Reports in Oncology}

\begin{tabular}{l|l}
\hline Case Rep Oncol 2017;10:795-801 \\
\hline DOI: 10.1159/000479224 & $\begin{array}{l}\text { @ 2017 The Author(s). Published by S. Karger AG, Basel } \\
\text { www.karger.com/cro }\end{array}$ \\
\hline
\end{tabular}

Montana et al.: Near Complete Response in a Patient with Classical Hodgkin Lymphoma Treated with Brentuximab Vedotin Concurrent with Radiation Therapy

6 Barlett, N, Chen R, Fanale M, et al: Retreatment with brentuximab vedotin in patients with CD30positive hematologic malignancies. J Hematol Oncol 2014;7:24.

-7 Chen R, Palmer J, Thomas SH, et al: Brentuximab vedotin enables successful reduced intensity allogeneic hematopoietic cell transplantation in patients with relapsed or refractory Hodgkin lymphoma. Blood 2012;119:6379-6381.

$>8$ Gibb A, Jones C, Bloor A, et al: Brentuximab vedotin in refractory CD30+ lymphomas: a bridge to allogeneic transplantation in approximately one quarter of patients treated on a Named Patient Programme at a single UK center. Haematologica 2013;98:611-614.

9 Fanale M, Whiting NC, Neylon E, et al: Treatment strategies to optimize outcomes with brentuximab vedotin: management of common and rare toxicities. Target Oncol 2015;4:15.

$\$ 10$ Forero-Torres A, Bartlett NL, Berryman RB, et al: Extended treatment with brentuximab vedotin in patients with relapsed or refractory CD30-positive hematological malignancies. Leuk Lymphoma 2015;56:1151-1153.

11 Hirsch A, Vander Els N, Gomez EG, et al: Effect of ABVD chemotherapy with and without mantle or mediastinal irradiation on pulmonary function and symptoms in early stage Hodgkin's disease. J Clin Oncol 1996;14:1297-1305.

12 Younes A, Connors JM, Park SI, et al: Brentuximab vedotin combined with ABVD or AVD for patients with newly diagnosed advanced stage Hodgkin lymphoma: long term outcomes (abstract). ASH Annual Meeting, 2014.

13 Kumar A, Casulo C, Yaholom, et al: Brentuximab vedotin and AVD followed by involved site radiotherapy in early stage, unfavorable risk Hodgkin lymphoma. Blood 2016;128:1458-1464.

14 Younes A, Bartlett N, Leonard JP, et al: Brentuximab vedotin (SGN-35) for relapsed CD30-positive lymphomas. N Engl J Med 2010;363:1812-1821.

-15 Senter PD, Sievers EL: The discovery and development of brentuximab vedotin for use in relapsed Hodgkin lymphoma and systemic anaplastic large cell lymphoma. Nat Biotechnol 2012;30:631-637.

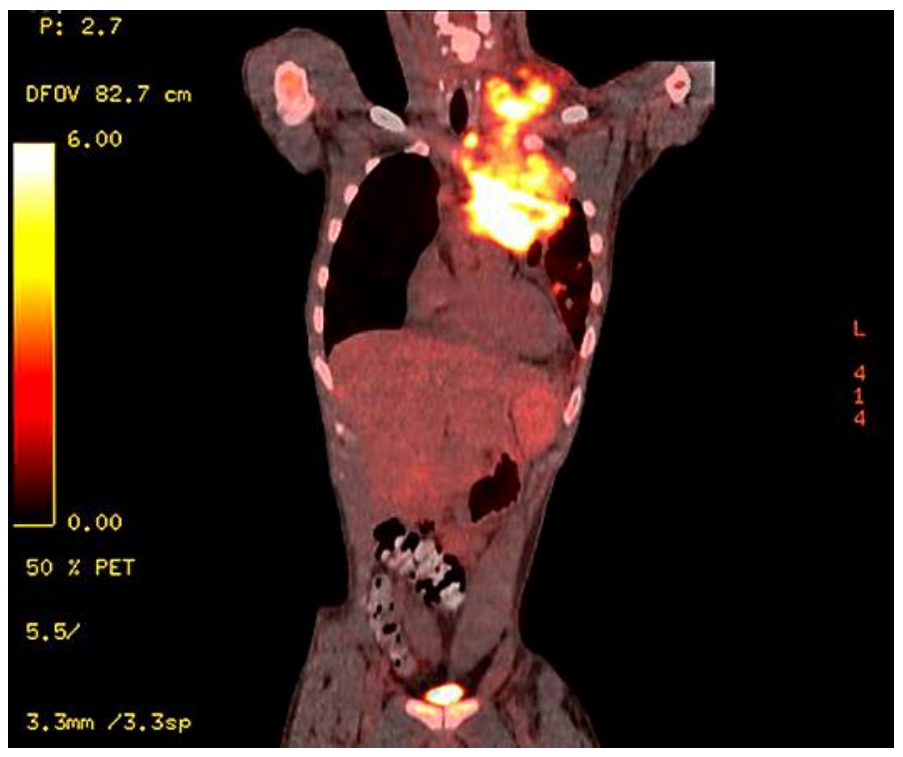

Fig. 1. Pretreatment PET/CT. 


\section{Case Reports in Oncology}

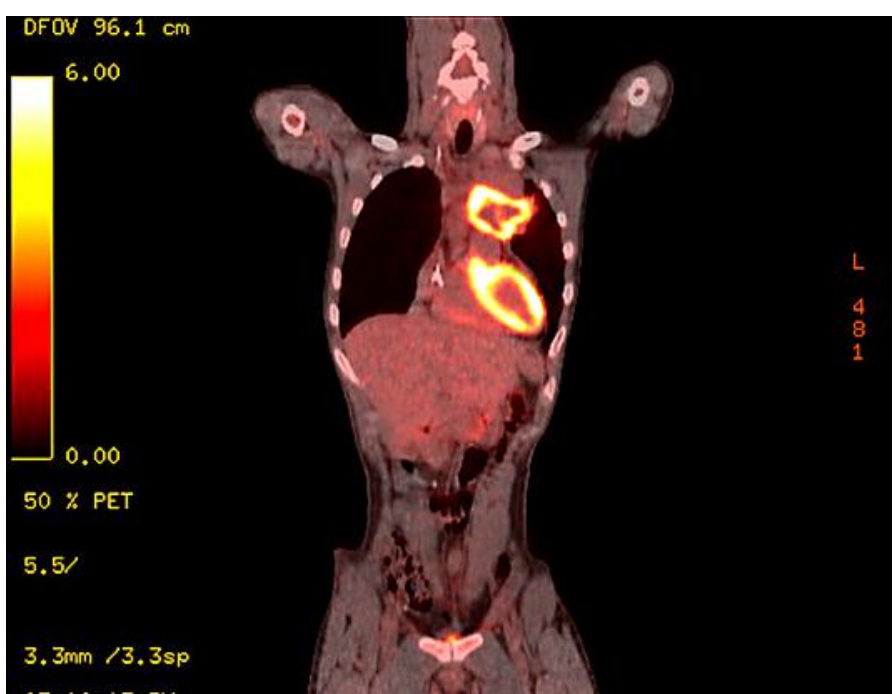

Fig. 2. PET/CT status after completion of 6 cycles of AVD (Adriamycin, vinblastine, dacarbazine) chemotherapy.

Montana et al.: Near Complete Response in a Patient with Classical Hodgkin Lymphoma Treated with Brentuximab Vedotin Concurrent with Radiation Therapy

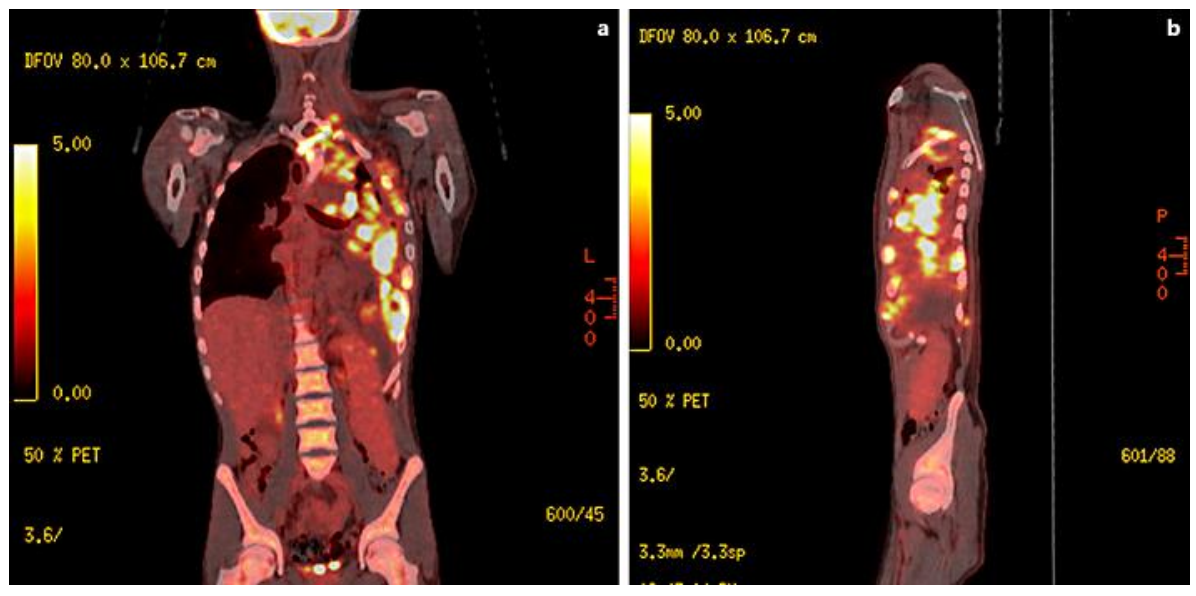

Fig. 3. a Coronal view of PET/CT status after 2 cycles of second-line ICE (ifosfamide, carboplatin, etoposide) chemotherapy showing residual and progressive disease. b Sagittal view of PET/CT status after 2 cycles of second-line ICE chemotherapy showing residual and progressive disease. 


\section{Case Reports in Oncology}

www.karger.com/cro

Montana et al.: Near Complete Response in a Patient with Classical Hodgkin Lymphoma Treated with Brentuximab Vedotin Concurrent with Radiation Therapy

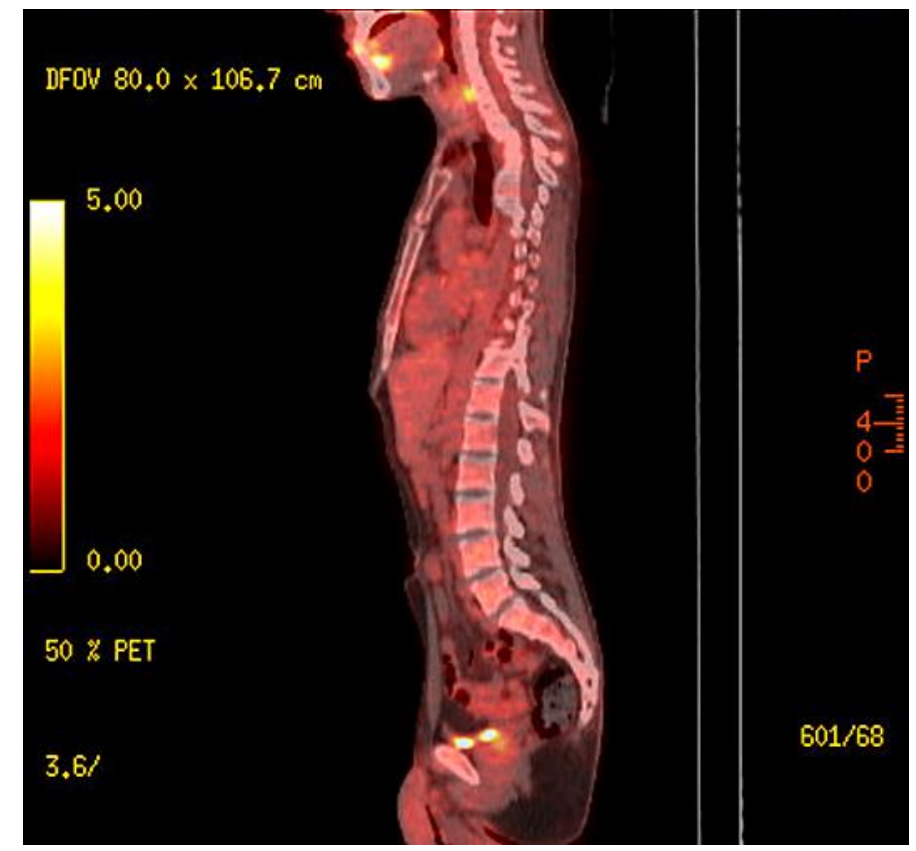

Fig. 4. PET/CT status after 4 cycles of brentuximab vedotin and concurrent radiation. 